Research Article

\title{
Heterogeneous Expression of Chondroitin Sulfate Glycosaminoglycans and Versican Proteoglycan during Early Development of Rathke's Pouch
}

\author{
Sheelah Iyengar and Anthony A. Capehart \\ Department of Biology, East Carolina University, N108 Howell Science Complex, Greenville, NC 27858, USA \\ Correspondence should be addressed to Anthony A. Capehart; capehartt@ecu.edu
}

Received 29 August 2014; Accepted 25 November 2014; Published 14 December 2014

Academic Editor: M. Michele Pisano

Copyright (C) 2014 S. Iyengar and A. A. Capehart. This is an open access article distributed under the Creative Commons Attribution License, which permits unrestricted use, distribution, and reproduction in any medium, provided the original work is properly cited.

\begin{abstract}
While much is known regarding morphogenetic factors involved in specification and differentiation of Rathke's pouch, less attention has been given to extracellular matrix (ECM) interactions involved in its formation. The present research investigated localization of two different chondroitin sulfate glycosaminoglycans (CS-GAGs), TC2 and d1C4, and versican CS-proteoglycan (PG) to identify additional ECM molecules involved in formation of the pituitary rudiment. Immunohistochemical evaluation of anterior pituitary primordia between $\mathrm{HH} 15$ and HH28 showed these ECM molecules prevalent in basement membrane and surrounding ECM underlying Rathke's epithelia and to a lesser extent between pouch epithelial cells. TC2/d1C4 CS-GAGs and versican showed changing and heterogeneous localization during pouch development that suggested specific roles in cell-ECM interaction during pituitary morphogenesis. TC2 antigen colocalized with versican at early stages in an asymmetric pattern, with particularly strong staining between ventral diencephalon and roof of Rathke's pouch while d1C4 CS-GAG encompassed the entire pouch by HH22 indicating association with a different CSPG. The heparan sulfate proteoglycan, perlecan, used to verify basement membrane structure, was a consistent component of Rathke's pouch. Data show a dynamic and heterogeneous pattern of CS-GAG and versican expression during early chick Rathke's pouch development that suggests new possibilities for ECM function in its establishment and growth.
\end{abstract}

\section{Introduction}

The pituitary gland is a small, lobed organ located at the base of the brain behind the optic chiasm in a concavity of the sphenoid bone. The anterior lobe or adenohypophysis derives from oral ectoderm and originates from Rathke's pouch [1], a hollow diverticulum that extends upwards from the stomodeum to underlie the diencephalon in the region of the infundibulum. An outpocketing of the infundibulum forms the primordium of the posterior lobe or neurohypophysis. In the chick, it has also been suggested that formation of Rathke's pouch may include a neuroectodermal contribution along with its stomodeal origin [2], with evagination and expansion toward the prechordal plate and anterior tip of the notochord underway by Hamburger and Hamilton stage 14 (HH14) [3]. Formation of lateral lobes at the lower end of Rathke's pouch by HH17/18 indicates beginning of the pars tuberalis and by HH25-28 developing glandular tissue of the pars distalis is evident during which time the proximal aspects of Rathke's pouch have become progressively constricted [4]. Over the next few days connection with the oral cavity deteriorates as the adenohypophysis continues glandular development. Synthesis of gonado- and thyrotropic hormones may be detected as early as HH25-29 and synthesis of other hormones follows shortly thereafter [5].

While much has been learned regarding the role of paracrine bone morphogenetic protein (BMP), fibroblast growth factor (FGF), sonic hedgehog (Shh), and Wnt signaling in activation of genes necessary for anterior pituitary commitment and formation [6-12], less attention has been given to the potential role of cell-extracellular matrix (ECM) interactions during morphogenesis of Rathke's pouch in avians or 
ECM involvement in pouch contact with the diencephalon. Early and pivotal studies of basement membrane laminin, collagen type IV, and fibronectin revealed close association with Rathke's pouch epithelium in the hamster and chick prior to glandular differentiation, providing suggestive evidence for an ECM role in pituitary development and maturation $[13,14]$. Basement membranes are well known as important structures that do much more than provide support for epithelia. Indeed, basement membranes have heterogeneous molecular composition and diverse roles dependent on tissue needs and regulate multiple functions including proliferation, migration, cell signaling, and differentiation [15].

Lectican family chondroitin sulfate proteoglycans (CSPGs) are important constituents of the ECM that are also selectively expressed in various embryonic basement membranes $[16,17]$ and function in numerous roles including matrix organization, growth, migration, and cell differentiation. One lectican CSPG, versican, has widespread distribution in the early embryonic ECM and its misexpression has been shown to disrupt cardiac [18], limb [19, 20], and joint development [20, 21]. Many CSPG functions are due to their complement of glycosaminoglycan (GAG) chains [22, 23] which may vary on a core protein dependent on tissue location and developmental stage. Heterogeneity of CS-GAGs in developing organs has been shown to correlate with key developmental events [17], suggesting that particular CSGAG structure (e.g., pattern and degree of sulfation) may be important in regulation of specific morphogenetic processes.

As few studies to date have identified ECM molecules expressed during early stages of anterior pituitary development $[13,14]$, the purpose of the present research was to investigate localization of two different CS-GAG antigens and the versican CSPG in association with Rathke's pouch to identify additional ECM molecules that may be involved in formation of this important pituitary rudiment. Data herein show a dynamic and heterogeneous pattern of CSGAG and versican expression during early chick Rathke's pouch development that suggests new possibilities for ECM function in its establishment and growth.

\section{Materials and Methods}

2.1. Antibodies. Monoclonal TC2 IgM antibody was generated by in vitro immunization of naïve mouse splenocytes with a nitrocellulose bound peanut agglutinin-positive fraction from micromass cultures of precartilage chick limb mesenchyme [24] and recognizes a native chondroitinasesensitive epitope on GAGs enriched in chondroitin-4-sulfate from bovine trachea (Sigma). The d1C4 monoclonal IgM was produced by in vitro immunization through direct exposure of naïve mouse splenocytes to unfixed micromass cultures of precartilage chick limb mesenchyme [16] and recognizes a native chondroitinase-sensitive epitope on GAG chains enriched in chondroitin-6-sulfate from shark cartilage (Sigma). Initial experiments utilized CS-56 mouse IgM (Sigma) which also recognizes a native CS-GAG epitope and staining patterns showing marked overlap with d1C4 localization in neighboring sections (not shown). Affinity purified polyclonal rabbit anti-chick versican IgG, directed against the whole native core protein, thus recognizing all isoforms [25], was a kind gift of Dr. Stanley Hoffman, Medical University of South Carolina. Anti-chick perlecan (clone 5C9) and anti-chick laminin (clone 3H11) mouse monoclonal IgGs were used to identify basement membrane structure and were obtained from the Developmental Studies Hybridoma Bank (University of Iowa, Iowa City, IA).

2.2. Immunohistochemistry. Chick embryos were fixed in $80 \%$ methanol $/ 20 \%$ dimethyl sulfoxide (Fisher), embedded in paraffin, and sectioned in sagittal orientation at $7 \mu \mathrm{m}$. Data presented herein for $\mathrm{HH} 15-28$ are representative of greater than 30 immunohistochemical experiments spanning developmental stages HH14/15, 17, 19/20, 22-25, 28, 35, and 38. Figures show adjacent sections from individual experiments immunostained within a $28 \mu \mathrm{m}$ range of tissue thickness.

Specimens were dewaxed in xylene, rehydrated through a graded ethanol series, washed in distilled water, and subjected to high temperature antigen retrieval using citrate buffer ( $\mathrm{pH}$ 6.0; Vector Labs) for 20 minutes [26]. Samples were allowed to cool, rinsed with distilled water, and subjected to $10 \mathrm{U} / \mathrm{mL}$ Streptomyces hyaluronidase (Sigma) for 1 hour at $37^{\circ} \mathrm{C}$ in $30 \mathrm{mM}$ sodium acetate, $\mathrm{pH} 5.2$, containing $125 \mathrm{mM}$ sodium chloride. Samples were washed thoroughly with phosphate buffered saline (PBS) before blocking 1 hour room temperature in 3\% bovine serum albumin (BSA, Fraction V, Sigma) and $1 \%$ normal goat serum (Sigma) in PBS. Specimens were incubated overnight at $4^{\circ} \mathrm{C}$ in $\mathrm{TC} 2, \mathrm{~d} 1 \mathrm{C} 4,5 \mathrm{C} 9$, and 3H11 hybridoma supernatants without dilution and antiversican was used at $2.6 \mu \mathrm{g} / \mathrm{mL}$. Double labeling of antiversican with TC2 was performed routinely. Sections were washed with PBS and incubated 2 hours at room temperature in $3.5 \mu \mathrm{g} / \mathrm{mL}$ FITC-conjugated-goat anti-mouse IgG or IgM (Cappel) or $7.0 \mu \mathrm{g} / \mathrm{mL}$ rhodamine-conjugated goat anti-mouse IgG or goat anti-rabbit IgG (Cappel) in blocking buffer. Samples were washed thoroughly in PBS, postfixed in $80 \% / 50 \%$ ethanol, equilibrated in PBS, and mounted in $10 \%$ PBS/90\% glycerol containing $100 \mathrm{mg} / \mathrm{mL}$ 1,4-diazabicyclo (2.2.2) octane (DABCO; Sigma). Controls for immunohistochemistry included omission of primary antibody or use of an irrelevant primary antibody. Slides were examined and imaged using an Olympus BX-40 equipped with epifluorescence optics and QColor5 camera and software.

General anatomical descriptions were based on Romanoff [27] and Bellairs and Osmond [28]. Reference to the cranial portion of Rathke's pouch adjacent to the ventral diencephalon as the roof and the caudal component continuous with oral ectoderm as the floor was used to maintain consistency with earlier published descriptions of pouch morphology $[2,13]$. Reference to the proximal pouch is that portion closer to the oral cavity and distal pouch refers to the pouch apex. All figures were oriented with diencephalon to the reader's left and stomodeum/oral cavity toward the bottom of each panel.

\section{Results}

3.1. Stage 15. In early Rathke's pouch at HH15 (Figure 1) CSGAG antigens enriched in chondroitin-6-sulfate (d1C4) and 


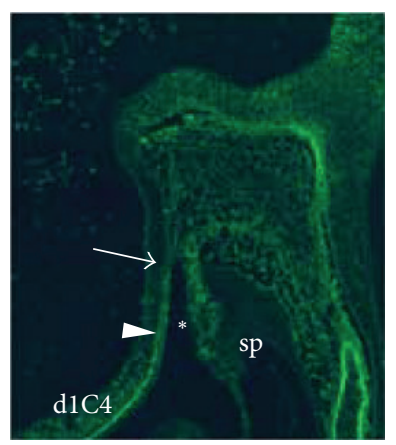

(a)

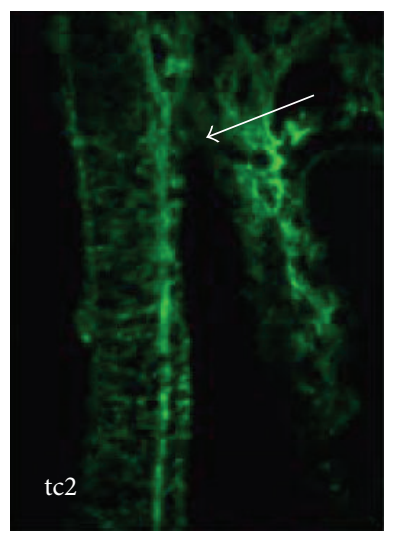

(e)

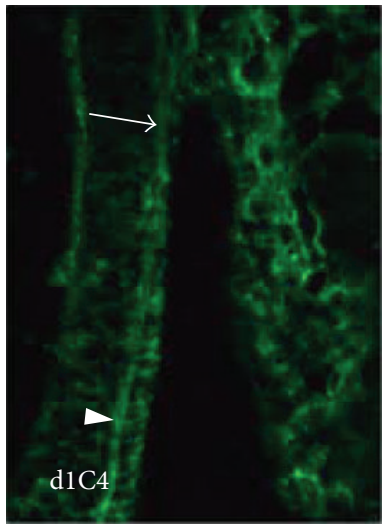

(i)

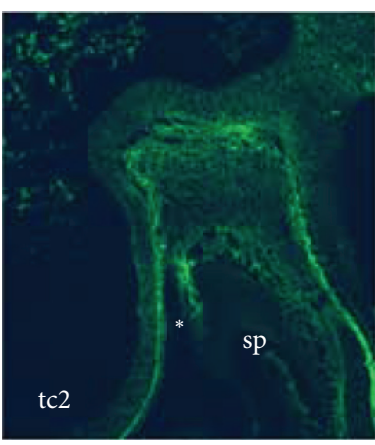

(b)

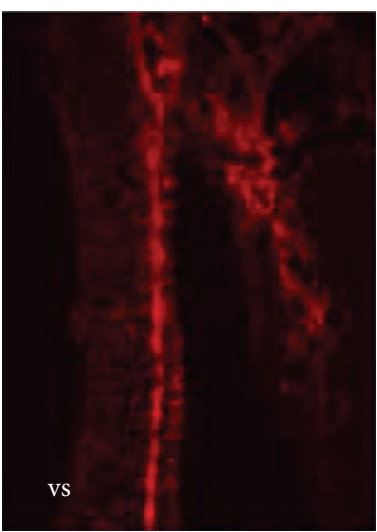

(f)

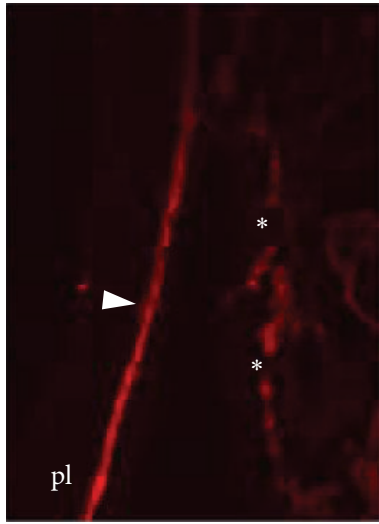

(j)

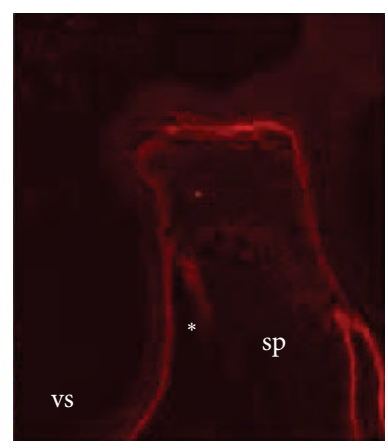

(c)

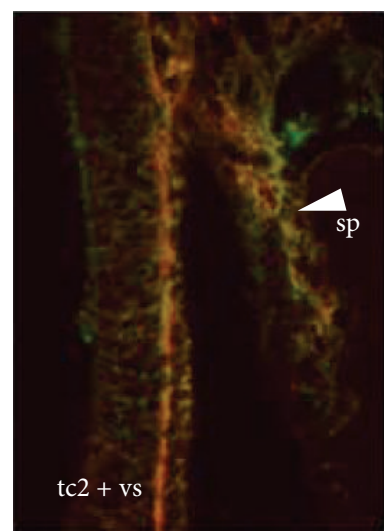

(g)

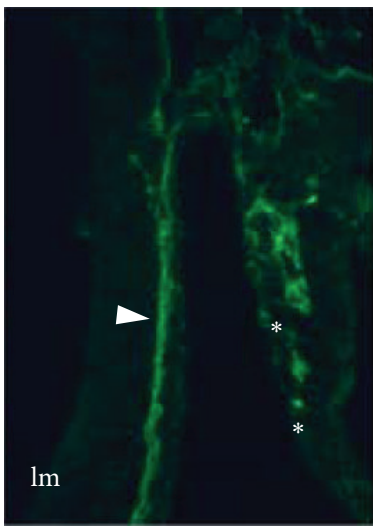

(k)

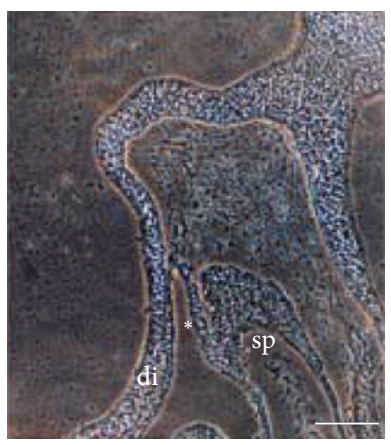

(d)

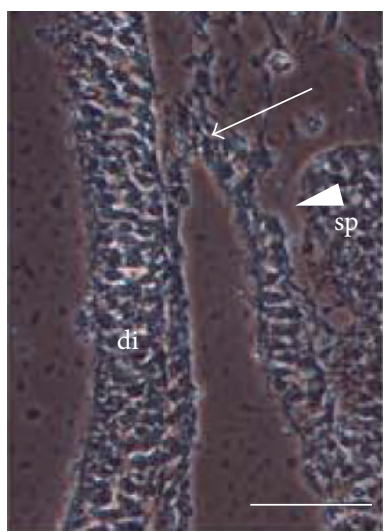

(h)

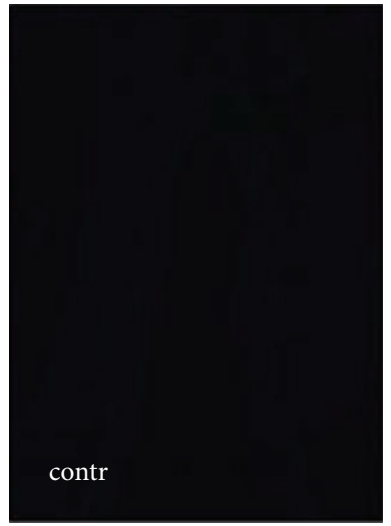

(l)

FIGURE 1: Sagittal sections showing CS-GAG and versican localization in the HH15 Rathke's pouch. (a) Low magnification of d1C4 CS-GAG associated with Rathke's pouch (small asterisk, pouch lumen). Note consistent pattern underlying diencephalon with gradual reduction in staining along pouch roof (small arrowhead to small arrow). (b) TC2 CS-GAG shows strong localization along the entire pouch roof and in the ECM of the distal half of the pouch floor adjacent to Seessel's pouch (sp) endoderm. (c) Versican CSPG (vs) shows a pattern like that of TC2. (d) Phase contrast image of (b) and (c) showing relationship of Rathke's pouch to neuroectoderm of the diencephalon (di) and Seessel's pouch. (e) Higher magnification image of TC2 antigen localization. Note reduced staining in ECM along distal tip of Rathke's pouch (large arrow). (f) Versican shows localization like that of TC2. (g) Overlay of versican and TC2 double labeled specimen shows colocalization of immunoreactivity. Note strong signal in ECM separating Rathke's from Seessel's pouch (large arrowhead). (h) Phase contrast image of (eg). (i) Higher magnification of d1C4 CS-GAG shows attenuation of staining along the roof of Rathke's pouch. Note intercellular staining of pouch epithelium of roof. (j) Perlecan (pl) demarcates basement membrane associated with Rathke's pouch (small arrowhead). Note gaps in basement membrane structure along pouch floor and tip (large asterisks). (k) Laminin $(\mathrm{lm})$ localization in the basement membrane of Rathke's pouch also showing interruptions in pouch floor and tip. (l) Representative immunohistochemical control (contr.) showing little or no specific immunoreactivity. Scale bar in (d) $=100 \mu \mathrm{m}(\mathrm{a}-\mathrm{d})$; (h) $=50 \mu \mathrm{m}(\mathrm{e}-\mathrm{l})$. 
chondroitin-4-sulfate (TC2) showed strong staining in the acellular ECM between the ventral diencephalon and roof of Rathke's pouch (Figures 1(a), 1(b), 1(d), and 1(i)). The d1C4 antigen showed reduced staining in the distal half of the pouch roof, but TC2 antigen was localized in a largely uninterrupted pattern along the length of the diencephalon. Staining was also noted for both CS-GAG antibodies between roof epithelial cells, with d1C4 antigen having a more consistent intercellular signal in the proximal portion. Along the Rathke's pouch floor (Figures 1(e), 1(f), 1(h), and 1(i)) TC2 and d1C4 antigens were expressed strongly in the matrix separating it from the anterior extent of Seessel's pouch endoderm. At sites of Rathke's pouch attachment to Seessel's pouch [2], TC2 reactivity was reduced, but $\mathrm{d} 1 \mathrm{C} 4$ antigen was still evident, though patchy. Weak d1C4 immunoreactivity was noted around the distal tip of Rathke's pouch, but little TC2 antigen was localized at this site.

Versican CSPG codistributed with TC2 CS-GAG in association with Rathke's pouch (Figures 1(c) and 1(f)) and double labeling showed strong colocalization along the pouch roof and floor (Figure $1(\mathrm{~g})$ ). Double labeling with d1C4 and antiversican core protein antibody showed overlap of signal in some sites but did not reveal the same close colocalization in Rathke's pouch as noted for TC2 (not shown).

Since CS-GAG and versican immunostaining of Rathke's pouch ECM appeared in several sites to be associated with basement membrane structure, immunostaining was also performed for two characteristic basement membrane constituents, laminin and perlecan. The heparan sulfate proteoglycan perlecan, which may sometimes be substituted with CS-GAG chains [29], codistributed closely with TC2 and versican (Figure $1(\mathrm{j})$ ), suggesting their association with basement membrane at sites such as the pouch roof. Laminin signal (Figure 1(k)) along the roof of Rathke's pouch was as reported previously [13] and similar to perlecan distribution. Along the pouch tip and floor both perlecan and laminin staining revealed a discontinuous basement membrane. Because of potential relevance to TC2 and $\mathrm{d} 1 \mathrm{C} 4 \mathrm{CS}-\mathrm{GAG}$ antigens as well as lack of information in general regarding perlecan association with Rathke's pouch development, antiperlecan was used for verification of basement membrane structure for the remainder of the study. Control specimens (Figure 1(1)) showed little or no staining at all stages examined.

\subsection{Stage 17. At HH17, midline TC2 CS-GAG expression} (Figures 2(a)-2(d)) again showed asymmetric distribution associated with Rathke's pouch with strong reactivity in the acellular matrix of the basement membrane of the pouch roof until reaching the infundibulum and reduced signal in the ECM along the floor that progressively diminished as the oral cavity was approached. Interestingly, again little TC2 staining occurred along the distal tip of Rathke's pouch which contacted the prechordal plate [2]. In slightly lateral sections, this pattern persisted and close colocalization of TC2 with versican CSPG was again observed (Figures 2(e)-2(h)). TC2 antigen and versican showed little expression at the pouch tip and reduced expression along the pouch floor. While intercellular staining was noted between neuroepithelial cells of the adjacent diencephalon, only weak signal within Rathke's pouch epithelium was noted. Pericellular matrix of nearby head mesenchyme was weakly positive for both.

The d1C4 CS-GAG displayed robust uniform staining along pouch roof and floor and in surrounding mesoderm. Staining was also evident between neuroectoderm cells of the diencephalon as well as between Rathke's pouch epithelial cells (Figures 2(i) and 2(j)). Like TC2 and versican, d1C4 localization was interrupted at the pouch tip.

Basement membrane perlecan overlapped with both CSGAGs and versican expression at sites surrounding Rathke's pouch. Perlecan localization showed that the basement membrane was continuous along the pouch floor at this stage but signal was also noticeably reduced at the pouch tip (Figure $2(\mathrm{k})$ ).

3.3. Stage 22. Dynamic patterns of CS-GAG and versican CSPG expression associated with Rathke's pouch continued into HH22 (Figure 3). TC2 antigen stained strongly underlying the ventral diencephalon particularly in the immediate region of the pouch roof where there was now a wider interval between roof and diencephalon. TC2 exhibited diminished signal underneath the infundibulum (Figures 3(a) and 3(b)). The pouch floor continued to display limited TC2 reactivity with exception of a small region of matrix toward its proximal aspect adjacent to developing glandular epithelium. Little staining of the distal pouch tip was apparent with TC2. The pericellular matrix of head mesenchyme surrounding Rathke's pouch stained positively and intercellular staining of neuroepithelial cells of the diencephalon was again noted. As in earlier stages versican CSPG colocalized closely with TC2 in Rathke's pouch, particularly along the roof, and also showed widespread staining of head mesenchyme ECM (Figures 3(c), 3(e), and 3(f)).

In contrast to TC2 and versican, d1C4 CS-GAG (Figures 3(d) and 3(g)) surrounded the length of Rathke's pouch roof, tip, and floor in a basement membrane-like pattern. Little signal was observed underneath the infundibulum. Weak d1C4 reactivity between cells of the pouch roof epithelium was present whereas pouch floor epithelial cells showed little intercellular localization. Staining of the apical surface of distal pouch cells was also present. Intercellular d1C4 antigen was noted within the diencephalon as was widespread pericellular staining of head mesenchyme around Rathke's pouch.

Perlecan clearly showed separation of basement membrane structure between the roof of Rathke's pouch and the diencephalon and revealed that the $\mathrm{d} 1 \mathrm{C} 4$ antigen was part of the ECM most closely associated with Rathke's pouch.

3.4. Stage 25. At HH25 proximal connection of Rathke's pouch to the oral cavity had undergone constriction. As at HH22, TC2 (Figures 4(a) and 4(b)) stained most heavily along the pouch roof and increased in intensity from its proximal aspect toward the pouch tip, which at this stage evidenced patchy expression of TC2. Weak TC2 CS-GAG expression along the tip continued beneath the floor in its distal third. Overall lighter staining was seen in Rathke's epithelia and along the apical edge of cells, particularly in more distal reaches of the floor, as was intercellular staining within the diencephalon above the distal pouch. At this stage versican 


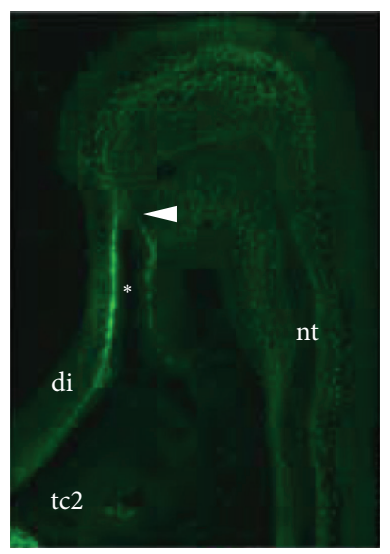

(a)

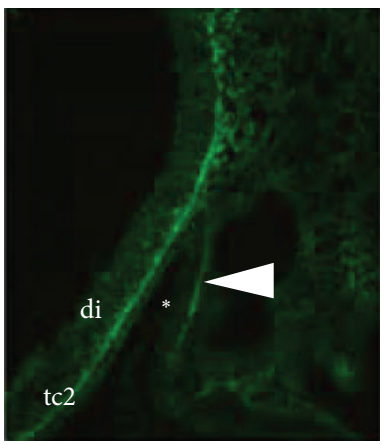

(e)

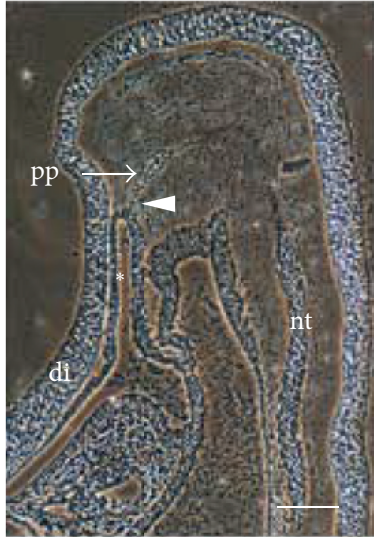

(b)

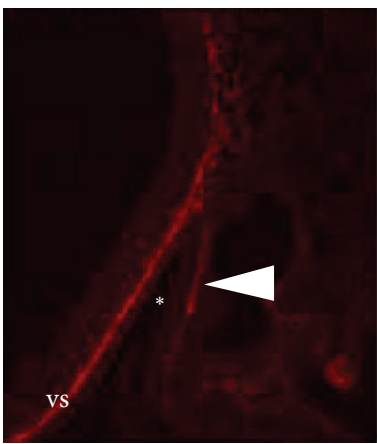

(f)

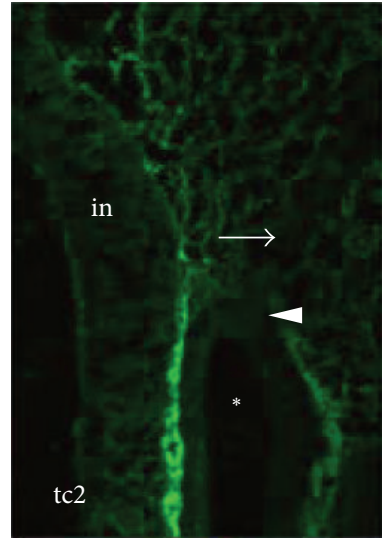

(c)

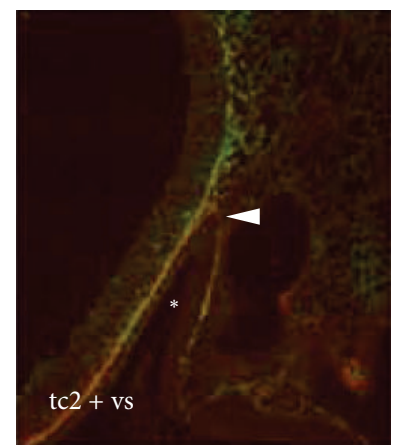

(g)

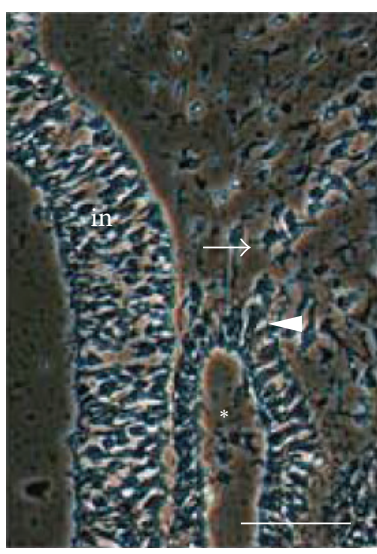

(d)

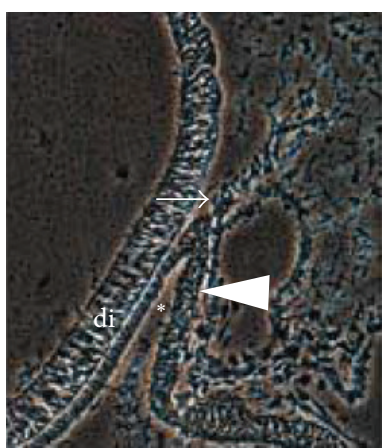

(h)

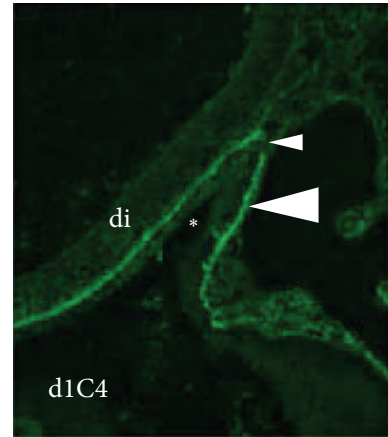

(i)

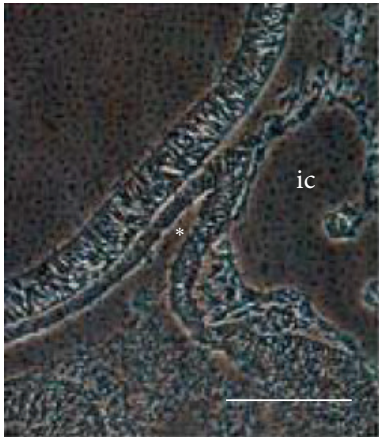

(j)

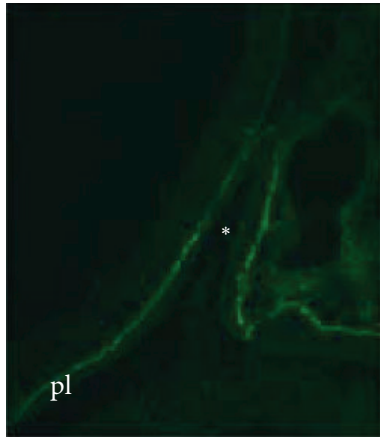

$(\mathrm{k})$

FIGURE 2: Sagittal sections showing CS-GAG and versican localization in Rathke's pouch at HH17. (a) Low magnification image of TC2 CSGAG localization associated with Rathke's pouch (asterisk denotes lumen). Strong reactivity is noted particularly along pouch roof underlying diencephalon (di). Note reduced staining along distal half of floor and at pouch tip (small arrowhead). (b) Phase contrast image of panel a shows prechordal plate (pp) following cranial flexure to Rathke's pouch anterior to notochord (nt). (c) Higher magnification of TC2 CS-GAG in panel a showing location of prechordal plate (small arrow). in: infundibulum. (d) Phase contrast image of (c). (e) TC2 and (f) versican (vs) double label shows colocalization surrounding Rathke's pouch. Note staining along distal pouch floor (large arrowheads). (g) Overlay of panels (e and f) highlights colocalization. Small arrowhead shows area of reduced TC2 and versican at tip of pouch. (h) Phase contrast image of panels (e-g). Note prechordal plate (small arrow). (i) d1C4 CS-GAG localization in Rathke's pouch. Note strong staining along entire perimeter of pouch including floor (large arrowhead) with exception of the distal pouch tip (small arrowhead). (j) Phase contrast image of (i). ic: internal carotid artery rudiment. (k) Perlecan ( $\mathrm{pl}$ ) highlights basement membrane structure associated with Rathke's pouch. Note lack of staining at pouch apex. Scale bar in (b) $=100 \mu \mathrm{m}$ (a and b); (d) $=50 \mu \mathrm{m}$ (c and d); (j) $=100 \mu \mathrm{m}$ (e-k).

expression (Figures 4(c) and 4(d)) began to clearly diverge from TC2 localization. There was ECM colocalization for much of the pouch roof; however, versican was largely absent from the distal pouch tip and also from the distal third of the floor that was enriched in the TC2 CS-GAG. Versican, however, was found underlying the floor in the proximal half of Rathke's pouch. TC2 and versican exhibited some overlap in the diencephalon but only little within epithelial cells of Rathke's pouch. As at earlier stages, versican stained pericellular matrix around head mesenchyme. 


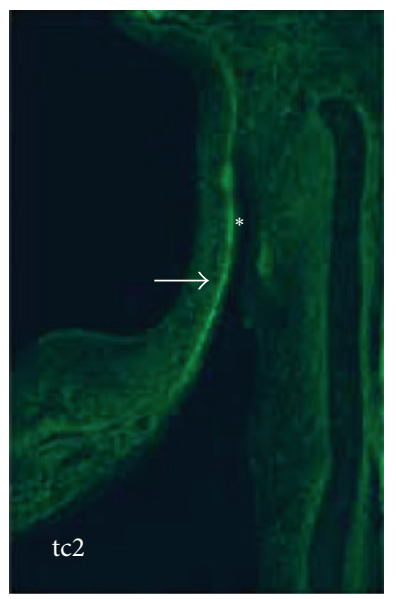

(a)

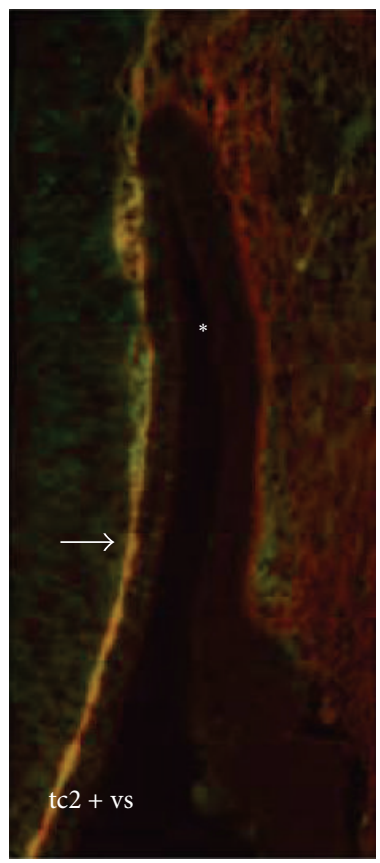

(e)

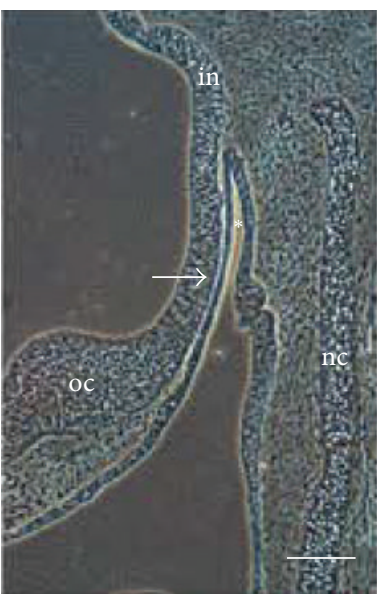

(b)

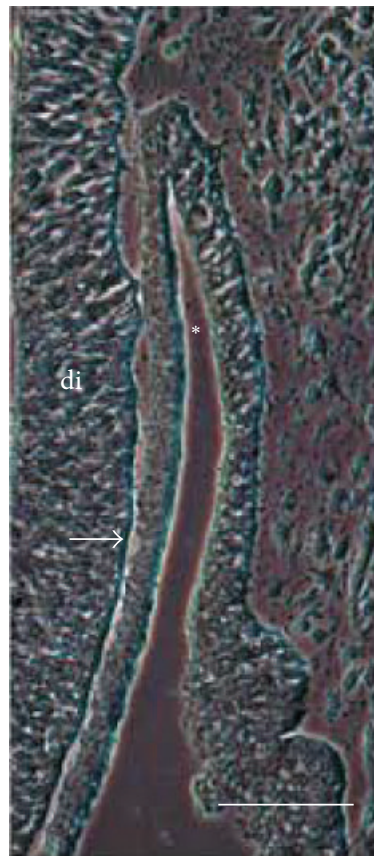

(f)

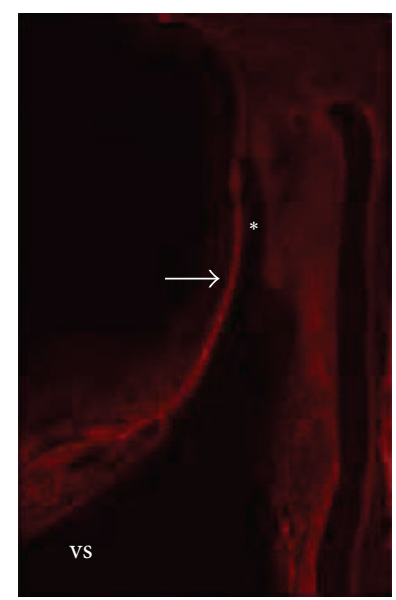

(c)

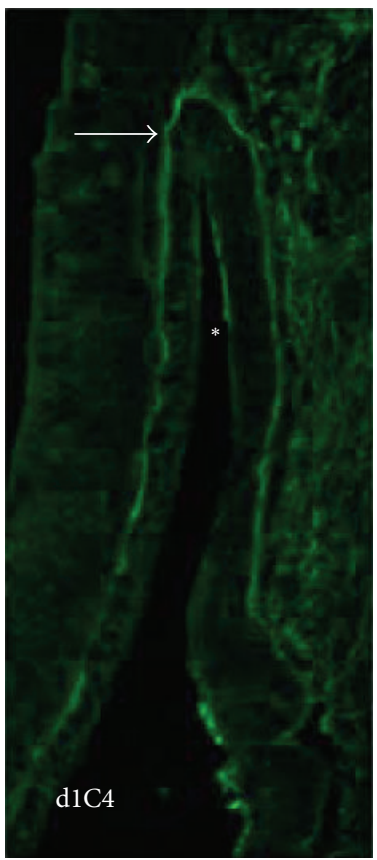

(g)

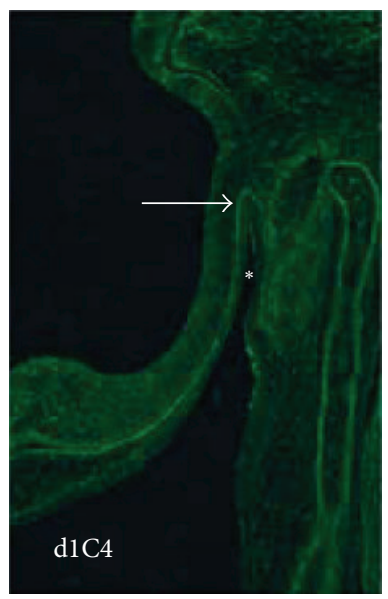

(d)

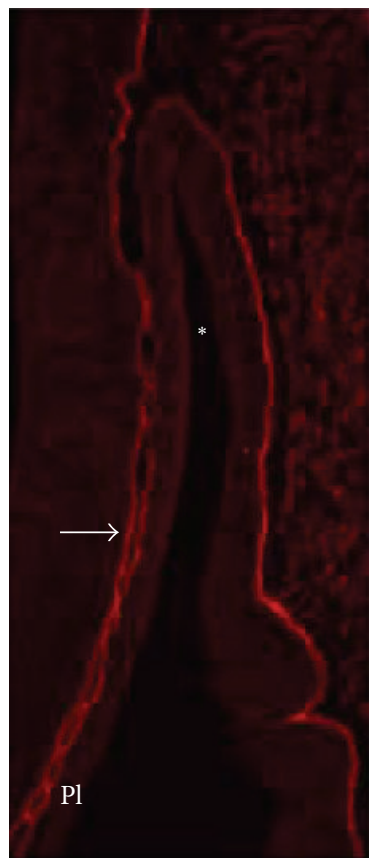

(h)

FIGURE 3: CS-GAG and versican localization in sagittal sections of Rathke's pouch at HH22. (a) Low magnification image of TC2 CS-GAG reveals Rathke's pouch associated staining along the pouch roof (small arrow; asterisk, lumen of Rathke's pouch). Note widespread reactivity with head mesenchyme and between neuroepithelial cells of diencephalon. (b) Phase contrast image of (a). in: infundibulum; oc: optic chiasm; nc: notochord. (c) Versican (vs) stains in a TC2-like pattern along the roof of Rathke's pouch (small arrow), head mesenchyme and within the diencephalon. (d) Low magnification of d1C4 shows reactivity in a basement membrane-like pattern around Rathke's pouch. Note staining encompassing entire tip of pouch (large arrow). (e) Double labeling of TC2 and versican shows colocalization associated with the roof of Rathke's pouch (small arrow) Note that versican and TC2 stain ECM throughout area between pouch roof and ventral diencephalon. (f) Phase contrast image of (e). di: diencephalon. (g) Higher magnification of d1C4 antigen expression surrounding Rathke's pouch, including pouch tip (large arrow). (h) Perlecan ( $\mathrm{pl}$ ) localization shows separate basement membrane structure associated with diencephalon and roof of Rathke's pouch (small arrow). Scale bar in (b) $=100 \mu \mathrm{m}(\mathrm{a}-\mathrm{d}) ;(\mathrm{f})=50 \mu \mathrm{m}(\mathrm{e}-\mathrm{h})$.

The $\mathrm{d} 1 \mathrm{C} 4$ antigen was also found along the pouch roof in a somewhat discontinuous basement membrane-like pattern though not so abundantly as for TC2 and versican (Figure 4(e)). Interestingly, like versican, d1C4 CS-GAG was not seen around the pouch tip and was largely absent from the distal pouch floor, but expression abruptly resumed in its proximal half. D1C4 staining was clearly observed within the diencephalon, but only weak staining was noted between cells of Rathke's pouch epithelium. Weak apical staining of epithelial cells in the distal aspect of the pouch was also seen.

Perlecan localization (Figure 4(f)) showed that basement membrane enshrouded Rathke's pouch although signal appeared slightly stronger in proximal and mid reaches of the pouch roof, where again separate, distinct basement 


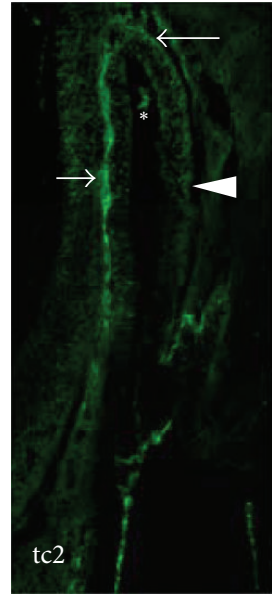

(a)

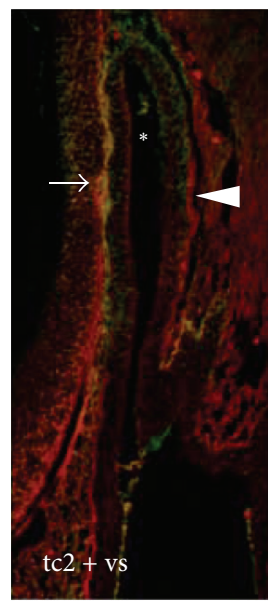

(d)

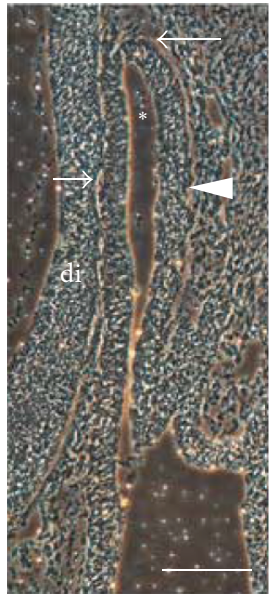

(b)

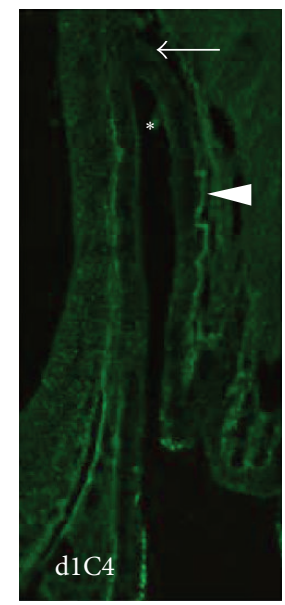

(e)

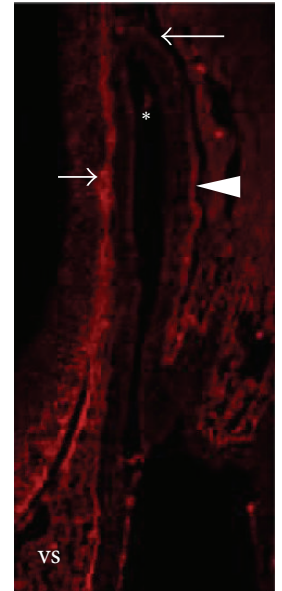

(c)

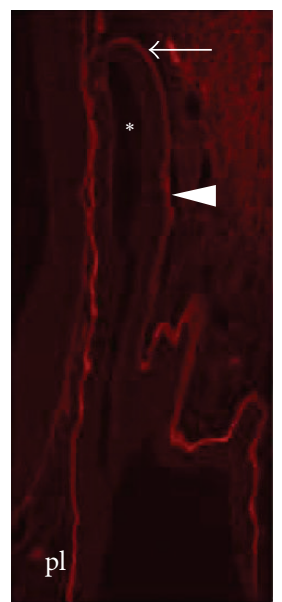

(f)

FIGURE 4: Sagittal sections showing CS-GAG and versican localization in Rathke's pouch at HH25. (a) TC2 CS-GAG stains ECM most strongly along the roof (small arrow) of Rathke's pouch (asterisk, lumen of Rathke's pouch) Note staining present around tip at this stage (large arrow) and along distal aspects of the pouch floor where it then abruptly ceases (arrowhead). (b) Phase contrast image of (a). Note constriction of proximal pouch lumen. di: diencephalon. (c) Versican (vs) codistributes with TC2 along pouch roof (small arrow), but little staining is seen around pouch tip (large arrow). Versican expression resumes along pouch floor (arrowhead) along its proximal aspect. (d) Overlay of TC2 and versican double labeled specimen. Note colocalization along pouch roof (small arrow), but areas of dissimilarity along pouch floor (arrowhead). (e) d1C4 CS-GAG stains in a discontinuous pattern in Rathke's pouch roof and floor. Note absence around pouch tip (large arrow) and staining underneath pouch floor in its proximal half (arrowhead). (f) Perlecan (pl) shows continuous basement membrane around Rathke's pouch including pouch tip (large arrow). Note less intense reactivity along pouch floor (arrowhead) than associated with roof. Scale bar in (b) $=100 \mu \mathrm{m}(\mathrm{a}-\mathrm{f})$.

membranes were apparent as distance increased between the diencephalon and roof of Rathke's pouch. Along the pouch floor perlecan staining was weaker and somewhat diffuse in appearance.

3.5. Stage 28. At $\mathrm{HH} 28$ cephalic and caudal lobes of the pars distalis were evident (Figure 5). TC2 CS-GAG staining (Figures 5(a) and 5(b)) was less pronounced in intensity relative to earlier stages and confined mostly to the roof of the caudal lobe and adjacent sparse mesenchyme beneath the ventral diencephalon. Intercellular staining was also evident between cells comprising this lobe, although signal was more prevalent in the roof epithelium. Staining along the apical surface of caudal lobe epithelia could also be seen. TC2 stained around the distal tip of the caudal lobe at point of contact with the infundibulum and underneath the lobe floor in its distal aspect. Little or no TC2 reactivity in the diencephalon was evident. TC2 CS-GAG in the cephalic lobe was largely confined to its perimeter and nearby mesenchyme, more so in its proximal aspect. Light staining between developing glandular cells of the cephalic lobe was seen as was weak intercellular staining in upper epithelial cells of the narrowed pouch connection to the oral cavity. Little overlap of TC2 antigen with versican was noted at this stage with exception of some peripheral staining around the cephalic lobe and along distal floor of the caudal lobe extending toward the infundibulum (Figures 5(c) and 5(d)). Weak versican staining between epithelial cells of the caudal lobe, 


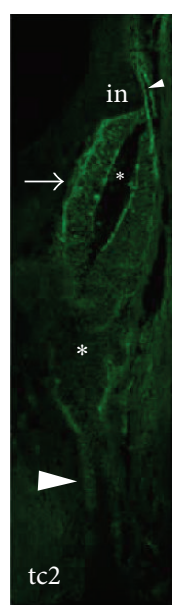

(a)

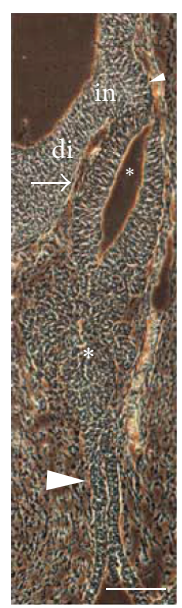

(b)

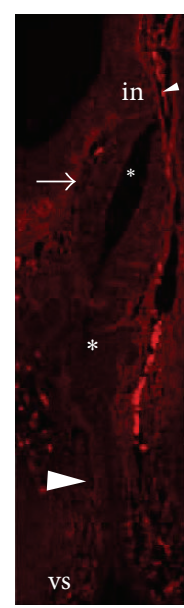

(c)

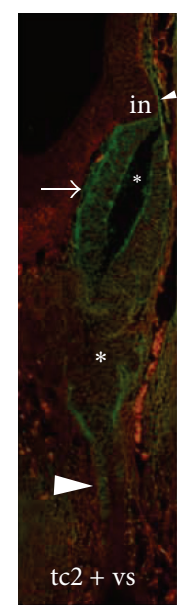

(d)

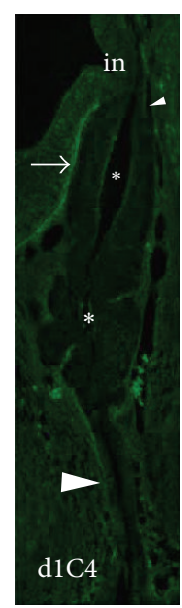

(e)

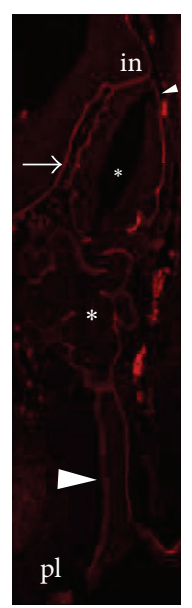

(f)

FIGURE 5: CS-GAG and versican localization in sagittal sections of Rathke's pouch at HH28. Note presence of cephalic (large asterisk) and caudal (small asterisk in lumen) lobes of the pars distalis. (a) TC2 CS-GAG is localized in mesenchyme and along the roof of the caudal lobe (arrow) and underneath the infundibulum (in, small arrowhead). Intercellular staining of roof cells of caudal lobe is also evident. Staining around cephalic lobe appears largely mesenchyme associated. Weak reactivity between cephalic lobe cells is seen as is weak staining between the narrowed Rathke's pouch connection to oral cavity (large arrowhead). (b) Phase contrast image of (a). di: diencephalon. (c) Versican (vs) is localized in diencephalon and is associated with surrounding head mesenchyme. Little staining is seen in roof of caudal lobe (arrow) although overlap with TC2 is noted along its distal floor extending toward infundibulum (small arrowhead). Weak reactivity between epithelia of the caudal lobe is seen. Note little staining associated with proximal connection of Rathke's pouch with oral cavity (large arrowhead). (d) TC2 antigen and versican double label shows limited colocalization associated with Rathke's pouch (arrow, arrowheads) but can clearly be seen in surrounding mesoderm. (e) d1C4 CS-GAG is localized within and beneath the diencephalon and along roof of the caudal lobe (arrow). Note reduced staining underlying infundibulum and distal floor of caudal lobe (small arrowhead). Weak staining is noted around cells of the cephalic lobe. Slight staining of epithelia and basement membrane underlying distal connection to oral cavity is present (large arrowhead). (f) Perlecan $(\mathrm{pl})$ demarcates basement membrane surrounding the pituitary rudiment. Note distinct basement membrane structures associated with diencephalon and caudal lobe (arrow, small arrowhead) and fusion at site of contact with infundibulum. Reduced perlecan expression can be seen associated with the roof side connection to oral cavity (large arrowhead). Scale bar in (b) $=100 \mu \mathrm{m}(\mathrm{a}-\mathrm{f})$.

particularly along the floor, was seen. Overall, Rathke's pouch associated versican immunoreactivity was much reduced at this stage, although head mesoderm and neuroepithelia of the diencephalon remained clearly positive.

Staining for the d1C4 CS-GAG was also much reduced relative to earlier stages with localization most prevalent along the roof of the caudal lobe similar to TC2 (Figure 5(e)). Staining was also evident underlying the ventral diencephalon and between neuroepithelia, although this was reduced in the infundibulum. D1C4 reactivity was also much decreased at point of contact between the caudal lobe and infundibulum. Staining along the floor of the caudal lobe and around the cephalic lobe appeared largely associated with surrounding mesodermal ECM. Light $\mathrm{d} 1 \mathrm{C} 4$ staining along the apical epithelial surface of the caudal lobe and intercellular staining between developing glandular cells of the cephalic lobe was also seen. Pouch connection to the oral cavity showed limited d1C4 staining along its edges and between pouch epithelia close to the oral cavity.

Perlecan (Figure 5(f)) again revealed basement membrane staining around the entire anterior pituitary rudiment as well as fusion of Rathke's pouch basement membrane with that of the infundibulum at point of contact. Interestingly perlecan stained less intensely along the narrowed pouch connection to the oral cavity along its roof.
At later stages of anterior pituitary development (e.g., HH38), staining for CS-GAGs and versican was reduced greatly with little TC2 CS-GAG or versican core protein immunoreactivity observed. The d1C4 CS-GAG exhibited areas of low level localization around the periphery of the gland that possibly represented early stages of connective tissue capsule formation [28] (not shown).

\section{Discussion}

The present study provided new information that at least two CS-GAG antigens and the versican CSPG are expressed during formation and early differentiation of Rathke's pouch. It also showed that these ECM molecules are prevalent in the basement membrane and immediately surrounding ECM underlying Rathke's epithelia and found to a lesser extent between cells of the pouch epithelium. Moreover, the TC2/d1C4 CS-GAGs and versican show changing localization during pouch development that suggests specific functions for these molecules in cell-ECM interactions during anterior pituitary morphogenesis. The heparan sulfate proteoglycan, perlecan, was also shown to be a consistent component of Rathke's pouch basement membrane.

At stages prior to HH25, the TC2 CS-GAG and versican colocalized closely in Rathke's pouch, suggesting that the TC2 
CS-GAG may be found on the versican core protein during early pouch formation as noted previously in endocardial cushion tissues $[18,24]$. The d1C4 CS-GAG overlapped with TC2 and versican at some sites and codistributed with versican along the proximal pouch floor at $\mathrm{HH} 25$; thus it is possible that a changing, developmentally regulated GAG complement on versican that included the $\mathrm{d} 1 \mathrm{C} 4$ antigen may occur during pituitary maturation. Clearly the diC4 CSGAG was also borne by one or more CSPGs other than versican at most stages examined, one likely candidate being the basement membrane CSPG, bamacan [29], although to our knowledge antibody to chick bamacan is not currently available commercially. D1C4 and TC2 CS-GAGs also codistributed with perlecan in discrete areas of basement membrane structure; thus it is possible that TC2 and $\mathrm{d} 1 \mathrm{C} 4$ epitopes could be found on perlecan at some sites since perlecan can bear chondroitin sulfate chains in addition to heparan sulfate [29].

Although versican and CS-GAG localization was noted in areas other than Rathke's pouch including ECM of head mesenchyme and neuroepithelium of the diencephalon, the basement membrane and associated ECM underlying Rathke's pouch epithelia was a major site of expression. Versican expression in the developing brain and its upregulation in glia during CNS injury has been well studied [30, 31], however, versican's potential role in adenohypophyseal development is not known, although it has been found previously as a constituent of basement membranes in several locations including heart, somites, notochord, neural tube, and vascular tissues $[24,32,33]$. It is possible that versican expression in and around Rathke's pouch may be regulated by Shh and/or canonical Wnt signaling [34], local factors present and necessary for early anterior pituitary formation (reviewed in [12]).

At early stages (HH15-22) versican and TC2 CS-GAG showed an asymmetric localization around Rathke's pouch relative to diC4 CAS-GAG and perlecan. Versican and TC2 expression was particularly strong in the basement membrane and intervening matrix between the diencephalon and Rathke's pouch epithelium. However, expression was essentially absent at the tip or apex of Rathke's pouch prior to HH25, particularly around site of contact with the prechordal plate at the earliest stages examined. This was an interesting observation since to our knowledge little is known of cell-matrix interactions between Rathke's epithelia and prechordal plate [2], adjacent head mesenchyme, or role in possible cell contact with the overlying neuroectoderm. Versican and TC2 localization appeared with decreasing intensity over time in the basement membrane and associated ECM in the distal portion of the pouch floor. The d1C4 CS-GAG on the other hand was initially incomplete in ECM surrounding Rathke's pouch and then "filled in" a basement membranelike pattern surrounding roof and floor of Rathke's epithelium by $\mathrm{HH} 22$. At $\mathrm{HH} 28 \alpha \mathrm{GSU}$, the common precursor to luteinizing hormone and thyroid stimulating hormone is highly expressed in the forming cephalic lobe of pars distalis [35] at which time versican, TC2, and $\mathrm{d1C} 4$ immunoreactivities appear reduced overall surrounding ECM as compared to earlier stages, although TC2 CS-GAG maintains expression in roof epithelia and adjacent mesenchyme of the caudal lobe. This pattern is different from that of versican and suggests association of TC2 antigen with another unidentified CSPG as noted earlier for $\mathrm{d} 1 \mathrm{C} 4$. To our knowledge, however, little is known at this time of potential CS-GAG or CSPG relationship to endocrine cell differentiation in vivo. The stage dependent dynamic heterogeneity in versican and CS-GAG expression documented in the present study raises the question as to why versican core protein and different CS-GAGs enriched in either chondroitin-4-sulfate or chondroitin-6sulfate are strategically expressed within and around the adenohypophyseal primordium.

Basement membranes are specialized, interacting ECM complexes comprised of several major proteins including collagen type IV, laminin, nidogen/entactin, and perlecan, supplemented by numerous other glycoproteins and proteoglycans that provide diverse biologic functions dependent on specific needs of epithelial tissues $[15,36]$. In addition to service as a supporting scaffold, basement membranes regulate proliferation, migration, cell signaling, and differentiation, many of these activities through integrin signaling [15]. Interestingly, the catalog of interactions and functions generally attributed to CSPGs and their CS-GAG chains are very similar to those of basement membranes $[22,23,37]$. Definitive understanding of versican function in adenohypophyseal development will require its conditional deletion in Rathke's epithelia as complete loss of functional versican in the $h d f$ mouse is embryonic lethal at an early stage due to severe cardiac defects [18]. However, the expression pattern of versican CSPG core protein and TC2/d1C4 CS-GAGs seen here suggest several interesting possibilities with regard to function. For example, versican may facilitate observed proliferation in the early pouch rudiment [35] through interaction of its G3 domain [37] with EGF receptors expressed by Rathke's pouch epithelium [38]. Antiadhesive cell-matrix interactions mediated by versican G1 domain binding to hyaluronan or G3 domain interference with fibronectinintegrin attachment [37] may aid elongation and extension of pouch structure. Alternatively, strong expression of versican/TC2 CS-GAG in the intervening matrix between the roof of Rathke's pouch and ventral diencephalon might simply serve a barrier function to prevent improper axonal migration [32].

A particularly interesting possibility for differential expression of CS-GAGs associated with versican and other CSPGs during anterior pituitary development is the potential for selective modulation of paracrine factor activities that regulate Rathke's pouch morphogenesis and differentiation. Recent studies have shown that different CS-GAGs can bind specific growth factors such as BMP, Fgf, and Shh with varying affinity $[22,39,40]$, enabling or inhibiting ligand delivery to receptors or sequestering needed factors in close proximity to responding cells. BMP, Fgfs, and Shh are all important in anterior pituitary commitment and differentiation and are expressed by the ventral diencephalon and other tissues such as Seessel's pouch endoderm or oral ectoderm $[9,10,12$, 35]. One possible function for CSPG expression lining the roof of Rathke's pouch could be to regulate levels of BMP4, Shh, and Fgf8 that reach receptors on Rathke's epithelia, 
as overexpression of these factors has been shown to cause pituitary hyperplasia $[9,10]$. This would likely involve cooperation of basement membrane heparan sulfate proteoglycans known to bind and regulate function of several growth factors including Fgfs and Shh [22]. Indeed, it has been shown that CS-GAG chains on perlecan can inhibit heparan sulfate-bound Fgf delivery to Fgf receptors [41]. Heterogeneity and changes in stage dependent expression observed in the present study with regard to TC2 and d1C4 CSGAG localization may reflect changing needs of Rathke's pouch epithelia to instructional information from inductive tissues such as the ventral diencephalon and infundibulum. Changing patterns of CS-GAG expression suggest the importance of specific carbohydrate structures to anterior pituitary development as observed with varying CS-GAG sulfation profiles for versican in the developing brain [42].

\section{Conclusions}

Results clearly provide new evidence that two CS-GAGs (TC2 and d1C4, enriched in chondroitin-4-sulfate and chondroitin-6-sulfate, resp.), the versican CSPG, and basement membrane perlecan are found associated with Rathke's pouch epithelium in the chick thus broadening the list of ECM molecules identified during early stages of adenohypophyseal development. CS-GAGs and versican were localized in basement membrane and surrounding ECM underlying Rathke's epithelia and also between epithelial cells of the pouch. Colocalization revealed that TC2 was likely found on the versican core protein during early stages of Rathke's pouch development while d1C4 CS-GAG was present on at least one other unidentified CSPG. CS-GAGs showed heterogeneous localization during pouch morphogenesis that suggests developmental regulation and specific roles for versican, TC2 and d1C4 antigens in cell-ECM interactions in anterior pituitary development.

\section{Conflict of Interests}

The authors declare that there is no conflict of interests regarding the publication of this paper.

\section{Acknowledgments}

The authors wish to thank Professor Stanley Hoffman for the generous contribution of the versican antibody used in this study.

\section{References}

[1] H. Rathke, "Uber die entstehung der glandula pituitaria," Archives of Anatomy and Physiology (Müller's Archives), vol. 5, pp. $482-485,1838$.

[2] A. G. Jacobson, D. M. Miyamoto, and S. H. Mai, "Rathke's pouch morphogenesis in the chick embryo," Journal of Experimental Zoology, vol. 207, pp. 351-366, 1979.

[3] V. Hamburger and H. Hamilton, "A series of normal stages in the development of the chick embryo," Journal of Morphology, vol. 8, pp. 241-245, 1951.
[4] W. J. Atwell and I. Sitler, "The early appearance of the anlagen of the pars tuberalis in the hypophysis of the chick," The Anatomical Record, vol. 15, no. 4, pp. 181-187, 2005.

[5] C. G. Scanes, L. E. Hart, E. Decuypere, and E. R. Kuhn, "Endocrinology of the avian embryo: an overview," Journal of Experimental Zoology, vol. 1, pp. 253-264, 1987.

[6] J. Ericson, S. Norlin, T. M. Jessell, and T. Edlund, "Integrated FGF and BMP signaling controls the progression of progenitor cell differentiation and the emergence of pattern in the embryonic anterior pituitary," Development, vol. 125 , no. 6, pp. 10051015, 1998.

[7] Y. Maruoka, N. Ohbayashi, M. Hoshikawa, N. Itoh, B. L. M. Hogan, and Y. Furuta, "Comparison of the expression of three highly related genes, Fgf8, Fgfl7 and Fgf18, in the mouse embryo," Mechanisms of Development, vol. 74, no. 1-2, pp. 175$177,1998$.

[8] M. Treier, A. S. Gleiberman, S. M. O’Connell et al., "Multistep signaling requirements for pituitary organogenesis in vivo," Genes and Development, vol. 12, no. 11, pp. 1691-1704, 1998.

[9] N. Takuma, H. Z. Sheng, Y. Furuta et al., "Formation of Rathke's pouch requires dual induction from the diencephalon," Development, vol. 125, no. 23, pp. 4835-4840, 1998.

[10] M. Treier, S. O'Connell, A. Gleiberman et al., "Hedgehog signaling is required for pituitary gland development," Development, vol. 128, no. 3, pp. 377-386, 2001.

[11] L. E. Olson, J. Tollkuhn, C. Scafoglio et al., "Homeodomainmediated beta-catenin-dependent switching events dictate celllineage determination," Cell, vol. 125, no. 3, pp. 593-605, 2006.

[12] X. Zhu, A. S. Gleiberman, and M. G. Rosenfeld, "Molecular physiology of pituitary development: signaling and transcriptional networks," Physiological Reviews, vol. 87, no. 3, pp. 933963, 2007.

[13] M. J. Horacek, J. C. Thompson, M. O. Dada, and L. Terracio, "The extracellular matrix components laminin, fibronectin, and collagen IV are present among the epithelial cells forming Rathke's pouch," Acta Anatomica, vol. 147, no. 2, pp. 69-74, 1993.

[14] R. E. Waterman and G. Balian, "Indirect immonufluorescent staining of fibronectin associated with the floor of the foregut during formation and rupture of the oral membrane in the chick embryo," Anatomical Record, vol. 198, no. 4, pp. 619-635, 1980.

[15] V. S. LeBleu, B. MacDonald, and R. Kalluri, "Structure and function of basement membranes," Experimental Biology and Medicine, vol. 232, no. 9, pp. 1121-1129, 2007.

[16] A. A. Capehart, M. M. Wienecke, G. T. Kitten, M. Solursh, and E. L. Krug, "Production of a monoclonal antibody by in vitro immunization that recognizes a native chondroitin sulfate epitope in the embryonic chick limb and heart," Journal of Histochemistry and Cytochemistry, vol. 45, no. 11, pp. 1567-1581, 1997.

[17] A. A. Capehart, J.-L. Scemama, C. A. Singhas, and S. Cox, "Heterogeneity of chondroitin sulfate glycosaminoglycan localization during early development of the striped bass (Morone saxatilis)," Anatomical Record, vol. 268, no. 1, pp. 47-58, 2002.

[18] C. H. Mjaatvedt, H. Yamamura, A. A. Capehart, D. Turner, and R. R. Markwald, "The Cspg2 gene, disrupted in the hdf mutant, is required for right cardiac chamber and endocardial cushion formation," Developmental Biology, vol. 202, no. 1, pp. 56-66, 1998.

[19] J. B. Shepard, D. A. Gliga, A. P. Morrow, S. Hoffman, and A. A. Capehart, "Versican knock-down compromises chondrogenesis in the embryonic chick limb," Anatomical Record, vol. 291, no. 1, pp. 19-27, 2008. 
[20] K. Choocheep, S. Hatano, H. Takagi, H. Watanabe, K. Kimata, and P. Kongtawelert, "Versican facilitates chondrocyte differentiation and regulates joint morphogenesis," The Journal of Biological Chemistry, vol. 285, no. 27, pp. 21114-21125, 2010.

[21] P. S. Nagchowdhuri, K. N. Andrews, S. Robart, and A. A. Capehart, "Versican knockdown reduces interzone area during early stages of chick synovial joint development," Anatomical Record, vol. 295, no. 3, pp. 397-409, 2012.

[22] L. Zhang, "Glycosaminoglycan (GAG) biosynthesis and GAGbinding proteins," Progress in Molecular Biology and Translational Science, vol. 93, pp. 1-17, 2010.

[23] R. V. Iozzo, "Matrix proteoglycans: from molecular design to cellular function," Annual Review of Biochemistry, vol. 67, pp. 609-652, 1998.

[24] A. A. Capehart, C. H. Mjaatvedt, S. Hoffman, and E. L. Krug, "Dynamic expression of a native chondroitin sulfate epitope reveals microheterogeneity of extracellular matrix organization in the embryonic chick heart," The Anatomical Record, vol. 254, pp. 181-195, 1999.

[25] M. K. B. Zanin, J. Bundy, H. Ernst, A. Wessels, S. J. Conway, and S. Hoffman, "Distinct spatial and temporal distributions of aggrecan and versican in the embryonic chick heart," The Anatomical Record, vol. 256, pp. 366-380, 1999.

[26] H. E. Snow, L. M. Riccio, C. H. Mjaatvedt, S. Hoffman, and A. A. Capehart, "Versican expression during skeletal/joint morphogenesis and patterning of muscle and nerve in the embryonic mouse limb," The Anatomical Record Part A: Discoveries in Molecular, Cellular, and Evolutionary Biology, vol. 282, no. 2, pp. 95-105, 2005.

[27] A. Romanoff, The Avian Embryo, The Macmillan Company, New York, NY, USA, 1960.

[28] R. Bellairs and M. Osmond, The Atlas of Chick Development, Academic Press, New York, NY, USA, 1998.

[29] J. R. Couchman, R. Kapoor, M. Sthanam, and R.-R. Wu, "Perlecan and basement membrane-chondroitin sulfate proteoglycan (bamacan) are two basement membrane chondroitin/dermatan sulfate proteoglycans in the Engelbreth-Holm-Swarm tumor matrix," Journal of Biological Chemistry, vol. 271, no. 16, pp. 9595-9602, 1996.

[30] C. E. Bandtlow and D. R. Zimmermann, "Proteoglycans in the developing brain: new conceptual insights for old proteins," Physiological Reviews, vol. 80, no. 4, pp. 1267-1290, 2000.

[31] R. A. Asher, D. A. Morgenstern, M. C. Shearer, K. H. Adcock, P. Pesheva, and J. W. Fawcett, "Versican is upregulated in CNS injury and is a product of oligodendrocyte lineage cells," The Journal of Neuroscience, vol. 22, no. 6, pp. 2225-2236, 2002.

[32] R. M. Landolt, L. Vaughan, K. H. Winterhalter, and D. R. Zimmermann, "Versican is selectively expressed in embryonic tissues that act as barriers to neural crest cell migration and axon outgrowth," Development, vol. 121, no. 8, pp. 2303-2312, 1995.

[33] B. Bode-Lesniewska, M. T. Dours-Zimmermann, B. F. Odermatt, J. Briner, P. U. Heitz, and D. R. Zimmermann, "Distribution of the large aggregating proteoglycan versican in adult human tissues," Journal of Histochemistry \& Cytochemistry, vol. 44, no. 4, pp. 303-312, 1996.

[34] M. Rahmani, B. W. Wong, L. Ang et al., "Versican: signaling to transcriptional control pathways," Canadian Journal of Physiology and Pharmacology, vol. 84, no. 1, pp. 77-92, 2006.

[35] H. Takagi, K. Nagashima, M. Inoue, I. Sakata, and T. Sakai, "Detailed analysis of formation of chicken pituitary primordium in early embryonic development," Cell and Tissue Research, vol. 333, no. 3, pp. 417-426, 2008.
[36] P. D. Yurchenco, "Basement membranes: cell scaffoldings and signaling platforms," Cold Spring Harbor Perspectives in Biology, vol. 3, no. 2, pp. 1-12, 2011.

[37] Y. J. Wu, D. P. La Pierre, J. Wu, A. J. Yee, and B. B. Yang, "The interaction of versican with its binding partners," Cell Research, vol. 15, no. 7, pp. 483-494, 2005.

[38] Y. Iwai-Liao, S. Kumabe, M. Takeuchi, and Y. Higashi, "Immunohistochemical localisation of epidermal growth factor, transforming growth factor $\alpha$ and EGF receptor during organogenesis of the murine hypophysis in vivo," Okajimas Folia Anatomica Japonica, vol. 76, no. 6, pp. 291-302, 2000.

[39] T. Miyazaki, S. Miyauchi, A. Tawada, T. Anada, S. Matsuzaka, and O. Suzuki, "Oversulfated chondroitin sulfate-E binds to BMP-4 and enhances osteoblast differentiation," Journal of Cellular Physiology, vol. 217, no. 3, pp. 769-777, 2008.

[40] D. M. Whalen, T. Malinauskas, R. J. C. Gilbert, and C. Siebold, "Structural insights into proteoglycan-shaped Hedgehog signaling," Proceedings of the National Academy of Sciences of the United States of America, vol. 110, no. 41, pp. 16420-16425, 2013.

[41] S. M.-L. Smith, L. A. West, P. Govindraj, X. Zhang, D. M. Ornitz, and J. R. Hassell, "Heparan and chondroitin sulfate on growth plate perlecan mediate binding and delivery of FGF-2 to FGF receptors," Matrix Biology, vol. 26, no. 3, pp. 175-184, 2007.

[42] H. Kitagawa, K. Tsutsumi, Y. Tone, and K. Sugahara, "Developmental regulation of the sulfation profile of chondroitin sulfate chains in the chicken embryo brain," The Journal of Biological Chemistry, vol. 272, no. 50, pp. 31377-31381, 1997. 

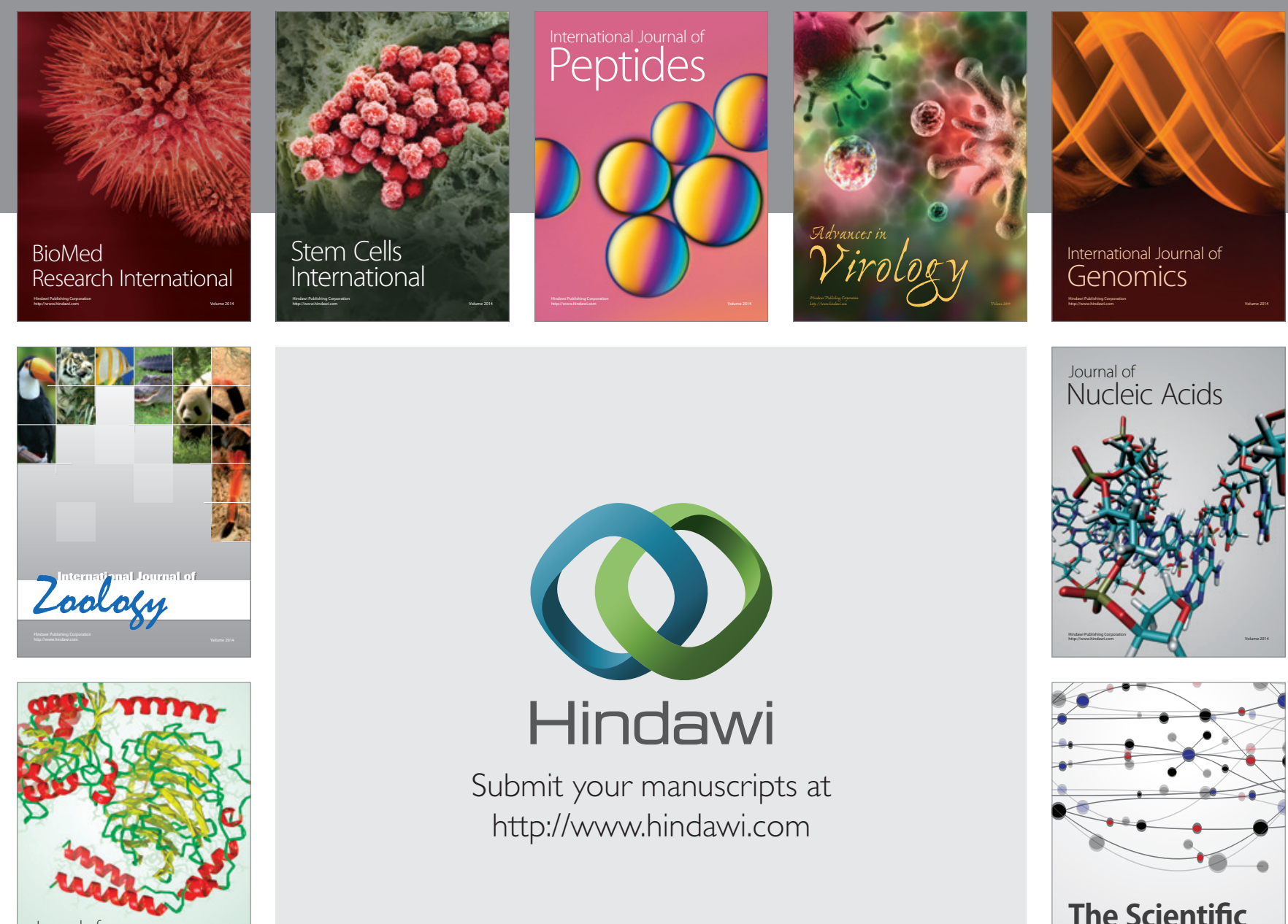

Submit your manuscripts at

http://www.hindawi.com

Journal of
Signal Transduction
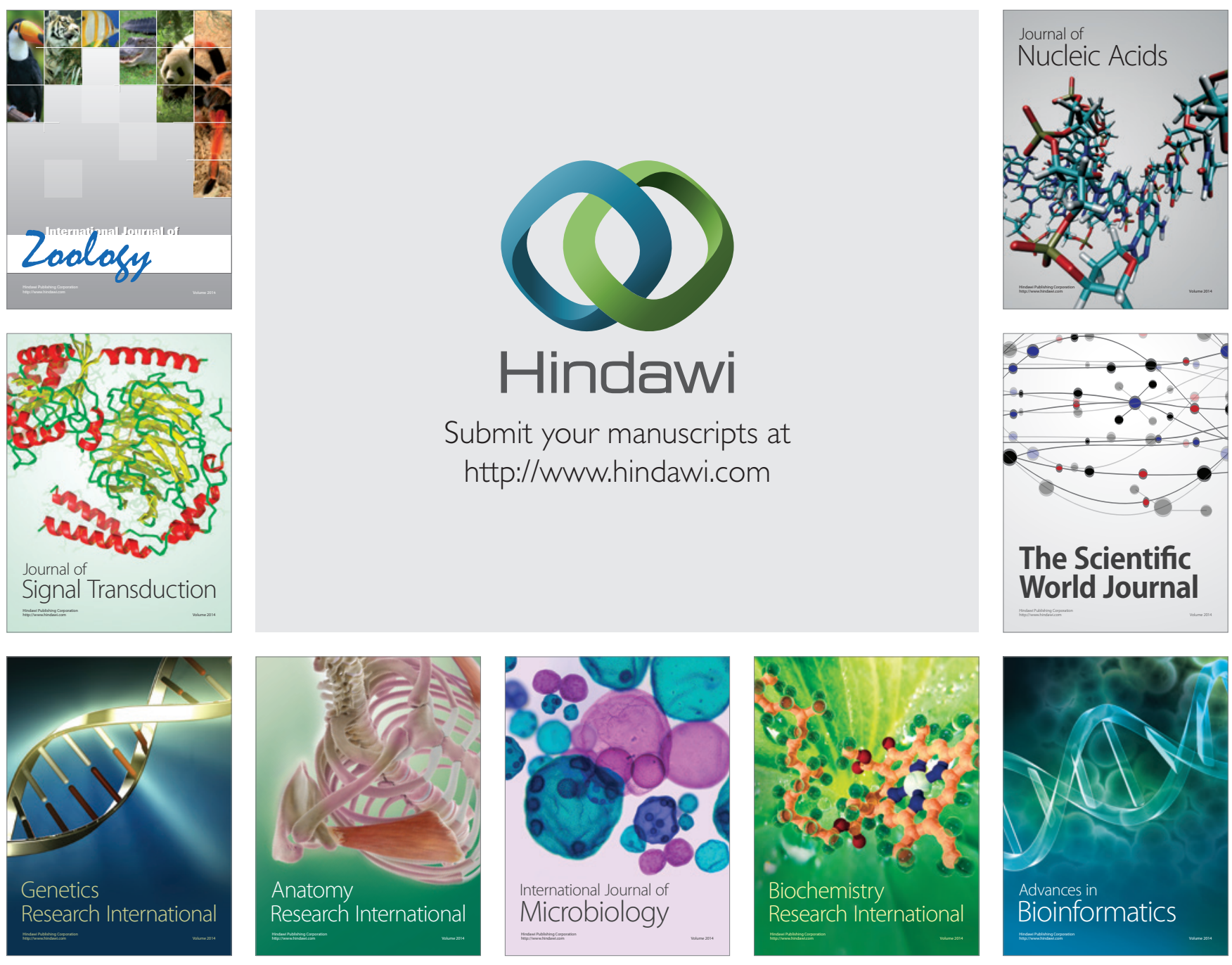

The Scientific World Journal
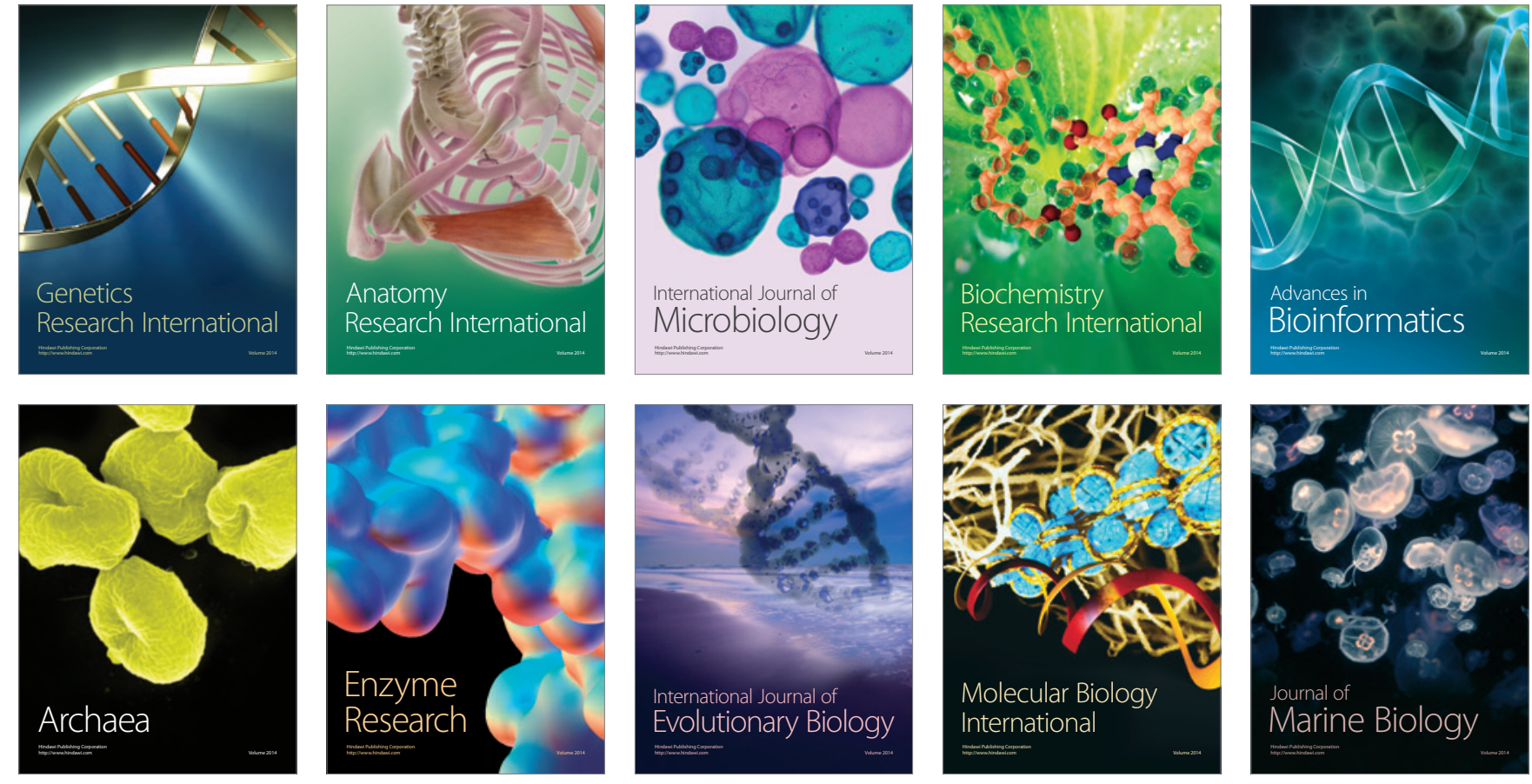